\title{
Applications of the Cesium Low Temperature Ion Source
}

Adam Steele $^{1}$, Andrew Schwarzkopf ${ }^{1}$ and Brenton Knuffman ${ }^{2}$

${ }^{1}$ zeroK NanoTech Corporation, United States, ${ }^{2}$ zeroK NanoTech Coporation, United States

We present results from FIB and FIB+SIMS systems featuring the $\mathrm{Cs}^{+}$Low Temperature Ion Source (LoTIS). When compared with other ion sources LoTIS can deliver very small spot sizes, high sputter rates, high yields of secondary ions, and a wide range of beam currents from pA to many nA.

We will review applications of LoTIS tested on a so-called FIB:ZERO platform. These applications include high resolution imaging, long depth-of-focus imaging, successful circuit edit operations on $10 \mathrm{~nm}$ node integrated circuits, high-precision machining of gold, and demonstration of the high grain-visibility imaging in copper and steel offered by LoTIS.

Previously we reported spot sizes as small as $(2.1 \pm 0.2) \mathrm{nm}$ (one standard deviation) are observed with a $10 \mathrm{keV}, 1.0 \mathrm{pA}$ beam. Brightness values as high as $(2.4 \pm 0.1) \times 10^{7} \mathrm{~A} \mathrm{~m}-2 \mathrm{sr}-1 \mathrm{eV}-1$ are observed near $8 \mathrm{pA} \mathrm{[1].} \mathrm{The} \mathrm{measured} \mathrm{peak} \mathrm{brightness} \mathrm{is} \mathrm{over} 24$ times higher than the highest brightness observed in a Ga liquid metal ion source (LMIS), and thousands of times higher than other Cs+ ion sources. This system can generate beams exceeding $20 \mathrm{nA}$. LoTIS is composed of a several discrete stages that collect, compress, cool and finally photoionize a cesium atomic beam using lasers [2].

The talk will show initial results from a new high resolution FIB+SIMS hybrid system called SIMS:ZERO. This system is capable of high-resolution FIB operations while also providing a new material analysis information channel through the application of Secondary Ion Mass Spectrometry (SIMS) via a double-focusing mass-spectrometer add-on. When compared with SIMS add-ons for existing FIB systems the $\mathrm{Cs}^{+}$LoTIS included with SIMS:ZERO will generate orders or magnitude more secondary ions, enabling high higher sensitivities. Conversely, when compared with existing focusedbeam dynamic SIMS instruments, SIMS:ZERO can provide >100x more current into a given spot, greatly reducing analysis times and allowing for creation of elemental maps at the fundamental limits of the SIMS technique. The prototype system is being built in collaboration with the Luxembourg Institute of Science and Technology (LIST).

High-resolution Cs+ focused ion beam systems stand to deliver considerable benefits for microanalysis and SIMS. 


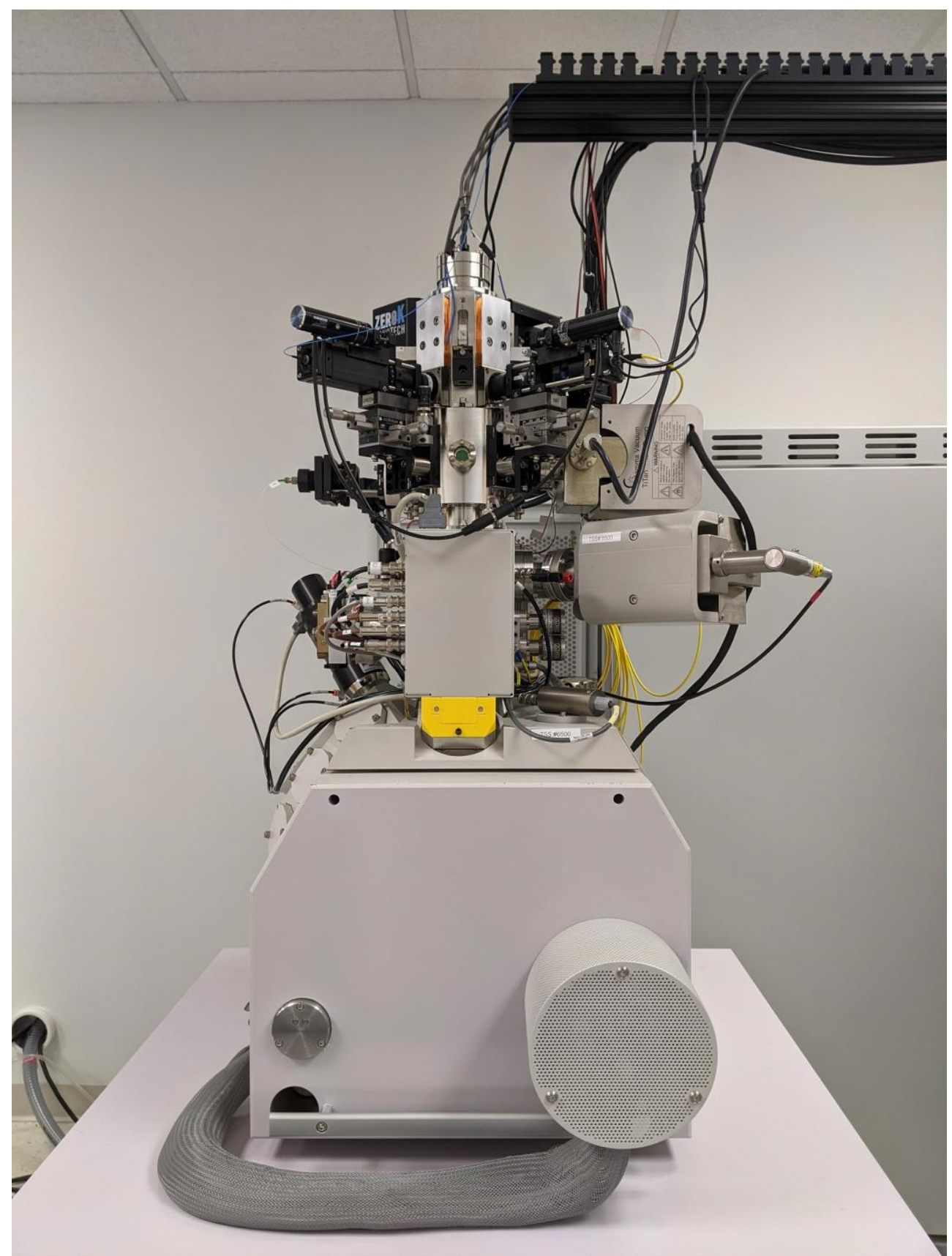

Figure 1. Cs LoTIS on a v600 FIB: zeroK's in-house FIB:ZERO is a retrofit of LoTIS to a Thermo-Fisher v600 platform. This upgrade improves the resolution to $<2.0 \mathrm{~nm}$ at $10 \mathrm{keV}$ and enables new contrast mechanisms. This system is also equipped with a platinum GIS. The SIMS spectrometer will be added in March 2021 to create the SIMS:ZERO platform.

\section{References}

[1] A. V. Steele, A. Schwarzkopf, J. J. McClelland, and B. Knuffman. Nano Futures. 1, 015005 (2017). [2] B Knuffman, AV Steele, and JJ McClelland. J. Appl. Phys. 114, 4 (2013). 